\title{
Mixed models for longitudinal left-censored repeated measures
}

\author{
Rodolphe Thiébaut $^{\mathrm{a}^{*}}$, Hélène Jacqmin-Gadda ${ }^{\mathrm{a}}$ \\ a INSERM E0338 Biostatistics, ISPED, Université Victor Segalen Bordeaux II, 146, rue Léo \\ Saignat 33076, Bordeaux Cedex, France
}

\section{Abstract}

Longitudinal studies could be complicated by left-censored repeated measures. For example, in Human Immunodeficiency Virus infection, there is a detection limit of the assay used to quantify the plasma viral load. Simple imputation of the limit of the detection or of half of this limit for left-censored measures biases estimations and their standard errors. In this paper, we review two likelihood-based methods proposed to handle left-censoring of the outcome in linear mixed model. We show how to fit these models using SAS® Proc NLMIXED and we compare this tool with other programs. Indications and limitations of the programs are discussed and an example in the field of HIV infection is shown.

Keywords: Mixed model, Repeated measures, Left-censoring, SAS proc NLMIXED, HIV infection

* Corresponding author. Tel: +33557574521 Fax: +33556240081

Email: rodolphe.thiebaut@ isped.u-bordeaux2.fr 


\section{Introduction}

Designs with repeated or grouped measures are common in epidemiological studies. The linear mixed model for correlated gaussian response is increasingly used, especially since availability of methods to fit such models in standard statistical packages like S-PLUS, BUGS or SAS [1-3].

However, longitudinal or grouped data could be complicated by left-censoring of some measures because of a detection limit of the assay used to quantify the marker. For example, this can occur with the concentration of some pollutants in environmental data [4], with antibody titre [5] or with Human Immunodeficiency Virus viral load in blood compartment (HIV RNA) [6, 7]. This latter example will be used throughout this paper because most of the methods to deal with left-censoring through longitudinal models have been proposed in this topic. HIV RNA ranged from 0 (in theory) to $6 \log _{10}$ copies/ml. The detection limit depends on the assay generation ranging from $4 \log _{10}$ copies/ml for the first assays available (in 1996) to 0.7 copies/ml today. Despite the improvement of the sensibility of assay, left-censoring is still an issue because antiretroviral treatments available since 1996 are very effective and lead to a steep decrease of HIV RNA after their initiation.

Several methods have been proposed to handle left-censoring of HIV RNA [6-11] rather than imputing the value of the detection limit or half of this limit. For mixed models, one can distinguish multiple imputation [8] and likelihood-based methods [6, 7, 10]. Among likelihood methods, Hughes [6] and Jacqmin-Gadda et al. [7] approaches differ only in the optimisation algorithm used to obtain maximum likelihood estimates. Lyles et al. have published an approach to deal with left-censoring of HIV RNA as well as informative dropout based on an hierarchical formulation of the likelihood [10]. Basically, all these methods lead to less biased estimates and increased standard errors of estimates compared to simple 
imputation methods. However, the diffusion of these methods could be limited by the use of Fortran $[6,7]$ or SAS® IML programs [10].

In this paper, we show how to use the new SAS® procedure NLMIXED [12] to fit mixed models taking into account left-censored repeated measures. We compare this procedure based on the conditional formulation of the likelihood given random effects with the algorithm proposed by Jacqmin-Gadda et al. [7] and based on a conditional formulation of the likelihood given observed measures. 


\section{Methods}

\subsection{Model}

We considered a linear mixed model [13] applied for modelling $\log _{10}$ HIV RNA. Let $Y_{i j}$, the $j^{\text {th }}$ measure at the time $t_{i j}\left(j=1, \ldots, n_{i}\right)$ for the subject $i(i=1, \ldots, N)$. For each subject $i$, we distinguished the $n_{i}^{o}$-vector of observed response $Y_{i}^{o}$ and the $n_{i}^{c}$-vector of censored response $Y_{i}^{c}$. A general formulation of the linear mixed model with $p$ explicative variables is:

$Y_{i}=X_{i} \beta+Z_{i} \gamma_{i}+e_{i}$ with $\left\{\begin{array}{c}e_{i} \sim N\left(0, \sigma_{e}^{2} I_{n_{i}}\right) \\ \gamma_{i} \sim N(0, G)\end{array}\right.$

where $X_{i}$ is a $n_{i} \times p$ design matrix, $\beta$ is a $p$-vector of fixed effects, $Z_{i}$ is a $n_{i} \times q$ design matrix which is usually a subset of $X_{i}, \gamma_{i}$ is a $q$-vector of individual random effects with $q \leq p$. Random effects and measurement errors $\left(e_{i}\right)$ are assumed to be independent.

\subsection{Likelihood}

Basically, two approaches are distinguished according to the development of the likelihood.

In Hughes [6] and Jacqmin-Gadda et al. [7] papers, the likelihood is formulated given $Y_{i}^{o}$ :

$$
\begin{aligned}
L(\theta) & =\prod_{i=1}^{N} f_{Y_{i}^{o} \mid \theta}\left(Y_{i}^{o} \mid \theta\right) \operatorname{Pr}\left(Y_{i}^{c}<c_{i} \mid Y_{i}^{o}, \theta\right) \\
& =\prod_{i=1}^{N} f_{Y_{i}^{o} \mid \theta}\left(Y_{i}^{o} \mid \theta\right) \iint_{H_{1} H_{2}} \ldots \int_{H_{n_{i}^{c}}} f_{Y_{i}^{c} \mid Y_{i}^{o}, \theta}\left(u \mid Y_{i}^{o}, \theta\right) d u_{1} d u_{2} \ldots d u_{n_{i}^{c}}
\end{aligned}
$$


with $\theta$ the vector of model parameters, $u$ a $n_{i}^{c}$-vector $\left[u_{1}, u_{2} \ldots u_{n_{i}^{c}}\right\rfloor^{T}$ and $\left.\left.H_{d}=\right]-\infty, c_{i d}\right]$ the range of integration with $d=1,2, \ldots, n_{i}^{c}$ and $c_{i}$ the $n_{i}^{c}$-vector of censoring threshold for the subject i. $f_{Y_{i}^{o} \mid \theta}(.$.$) is the multivariate normal density of observed measures and f_{Y_{i}^{c} \mid Y_{i}^{o}, \theta}(.$.$) is$ the multivariate normal conditional density of censored measures given observed measures. Thus, computation of this likelihood needs calculation of a multiple integral as large as the number of censored measured bysubject.

To use the NLMIXED procedure or the Lyles et al. program [10], the likelihood is formulated given the random effects:

$L(\theta)=\prod_{i=1}^{N}\left[\int_{R^{q}}\left\{\prod_{j=1}^{n_{i o}} f_{Y_{i j}^{o} \mid \gamma_{i}}\left(Y_{i j}^{o} \mid \gamma_{i}=u\right)\right\}\left\{\prod_{j=n_{i o}+1}^{n_{i c}} \phi_{Y_{i j}^{c} \mid \gamma_{i}}\left(Y_{i j}^{c} \mid \gamma_{i}=u\right)\right\} f_{\gamma_{i}}(u) d u_{1} d u_{2} \ldots d u_{q}\right]$

$f_{Y_{i j}^{o} \mid \gamma_{i}}(.$.$) is the univariate normal conditional density of the observed measure j$ in subject $i$ given random effects and $\phi_{Y_{i j}^{c} \mid y_{i}}(. \mid)$ is the univariate normal cumulative distribution function of the censored measure $j$ in subject $i$ given random effects. With this formulation, computation of this likelihood needs calculation of a multiple integral as large as the number of random effects included in the model. 


\subsection{Computation}

Whatever the method used, estimation of model parameters is based on maximum likelihood and need computation of multiple integral. However, the approaches differ in term of dimension of the integrals, numerical methods used to compute those integrals and optimisation algorithm used to maximise the likelihood.

The Fortran program proposed by Jacqmin-Gadda et al. [7] to maximise the likelihood (1) is called CENSAD and is available at http://www.isped.u-bordeaux2.fr. It is based on a Marquardt algorithm [14] that is a Newton-Raphson like algorithm where the diagonal of the Hessian matrix is inflated when adapted. To impose a positive constraint of covariance parameters, a new parameterisation of the model was used in term of squared root of $\sigma_{e}^{2}$ and a Cholesky decomposition of the random effects' covariance matrix. Multiple integrals of multivariate normal density as large as the number of censored measures $n_{i}^{c}$ were numerically calculated using a subregion adaptative multiple integration method [15]. This integration algorithm was found to be optimal when the size of the integral is less than 10 [16]. The algorithm developed by Hughes [6] aims also to maximise the likelihood (1) but it is based on an EM algorithm and a Gibbs sampler to compute the integral in the E step. Those two algorithms have been previously compared in a simulation study and it has been shown that the MCEM algorithm presents more convergence problems and leads to more biased estimates for some covariance structures [7].

The NLMIXED procedure is available since SAS ${ }^{\circledR}$ version 7 and has been written to fit non linear mixed model $[12,17]$. This procedure allows specifying a general form for the conditional distribution of the response variable given the random effects. Thus, a general log likelihood function (given the random effects) can be specified using the option general in the 
statement $\underline{\text { model }}$. Then, the procedure directly maximises the approximate integrated likelihood. Several optimisation algorithms can be used with NLMIXED through the option technique such as Conjugate Gradient, Double Dogleg, Nelder-Mead Simplex, classic, Ridge or Quasi Newton-Raphson. By default, NLMIXED performs a Quasi-Newton optimisation. This algorithm works with an approximation of the Hessian matrix and thus does not need calculating second order derivatives, which is computationally intensive. This great choice in algorithms allows using the optimal one according to the kind of data [18]. By default, the computation of the integral over random effects is performed by an adaptive Gaussian quadrature method [19] but other methods such as importance sampling (a Monte Carlo method) may be chosen using the option methods. The IML program proposed by Lyles et al. [10] and NLMIXED are very close in their approach. However, the IML program was written for a model including only a random intercept and a random slope and then need to be rewritten for another model. The program uses a simple quadrature method to integrate over random effects that is clearly weaker than the adaptative quadrature implemented in NLMIXED [20]. Moreover, computational time is definitely longer than other methods (about half an hour for the presented application). The advantage of IML is the potential of programming very different models and, for example handling informative dropout as proposed by Lyles et al. [10].

Differences between approaches are summarised in table 1. 


\section{Codes for NLMIXED}

\subsection{Univariate mixed model}

The model considered in the following included a fixed $(\alpha)$ and random $\left(a_{i}\right)$ intercept and a fixed $(\beta)$ and random $\left(b_{i}\right)$ slope:

$Y_{i j}=\alpha+a_{i}+\left(\beta+b_{i}\right) t_{i j}+e_{i j}$

with $G=\left[\begin{array}{ll}\sigma_{1}^{2} & \sigma_{12} \\ \sigma_{12} & \sigma_{2}^{2}\end{array}\right], e_{i j} \sim N\left(0, \sigma_{e}^{2}\right)$ and $\left[\begin{array}{c}a_{i} \\ b_{i}\end{array}\right] \perp e_{i}$

The code to fit such model accounting for left-censoring of $Y_{i j}$ is:

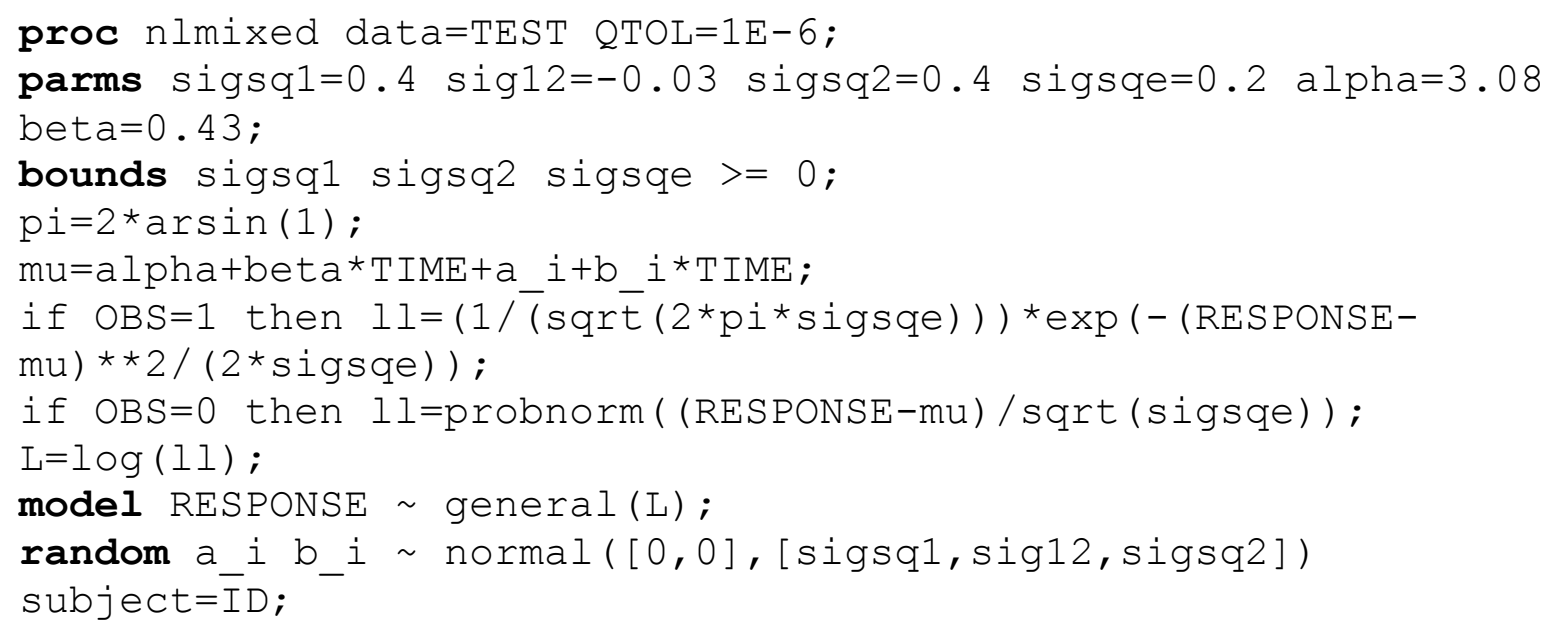

Model parameters must be declared in the first statement parms. Starting values are not mandatory but could be crucial in case of convergence difficulties. The statement $\underline{\text { bounds }}$ allow specifying some constraints on parameters. In the following example, variance parameters $\left(\sigma_{1}^{2}, \sigma_{2}^{2}\right.$ and $\left.\sigma_{e}^{2}\right)$ are constrained to be positive. The dependent variable named "RESPONSE" is declared to follow a general log-likelihood that returns the value $\mathrm{L}$ in the statement $\underline{\text { model }}$. The programming lines between the statements $\underline{\text { bounds }}$ and model define 
this log-likelihood given the random effects. The conditional distribution of $Y_{i j}$ given the random effects is defined by expectancy $\mu=E\left(Y_{i j} \mid a_{i}, b_{i}\right)=\alpha+\beta t_{i j}+a_{i}+b_{i} t_{i j}$ and the variance $\operatorname{Var}\left(Y_{i j} \mid a_{i}, b_{i}\right)=\sigma_{e}^{2}$. Then, according to the status of $Y_{i j}$, i.e. observed $(\mathrm{OBS}=1)$ or left-censored $(\mathrm{OBS}=0)$, the contribution to the likelihood is the density or the cumulative distribution function calculated by the function probnorm of a univariate normal variable. Because the procedure minimises the log-likelihood, the value of the likelihood contribution for the observation $Y_{i j}$ is log-transformed. The distribution for the random effects is specified in the statement random. Today, only normal distribution is available with NLMIXED. When defining the lower triangle of the random effects covariance matrix, some of covariance elements can be set to 0 . That could be useful to test a covariance with a likelihood ratio statistics. In the statement $\underline{\text { random, }}$, the option subject=id defined how the dataset is clustered. Nested random effects are not possible at present.

In case of difficulties for convergence, several options are available apart from changing starting points (statement parms) and algorithm (options technique, linesearch, update). For example, the option optcheck avoid terminating at a stationary point by checking if the likelihood around the convergence point is not better than the likelihood at convergence. The convergence criteria (based on likelihood function, parameters or gradient) can be modified. Sometimes, difficulties come from numerical calculation of integrals or derivatives. Precision or methods for these calculations are modifiable. For example, derivatives can be approximated using forward $(f d=$ forward $)$ or central differences $(f d=$ central $)$ and tolerance

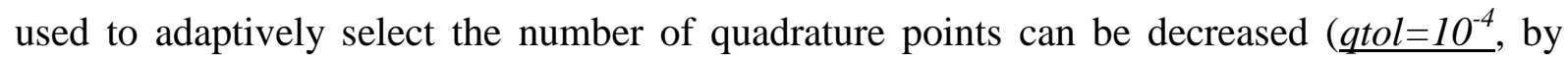
default).

The positive constraint on variance parameters $\left(\sigma_{1}^{2}, \sigma_{2}^{2}\right.$ and $\left.\sigma_{e}^{2}\right)$ is not enough to insure that the covariance $\mathrm{G}$ is positive definite. In the example, this constraint can be respected when 
formulating the constraint on the correlation coefficient. This leads to the same results in the example presented in results section. The code is modified as follow:

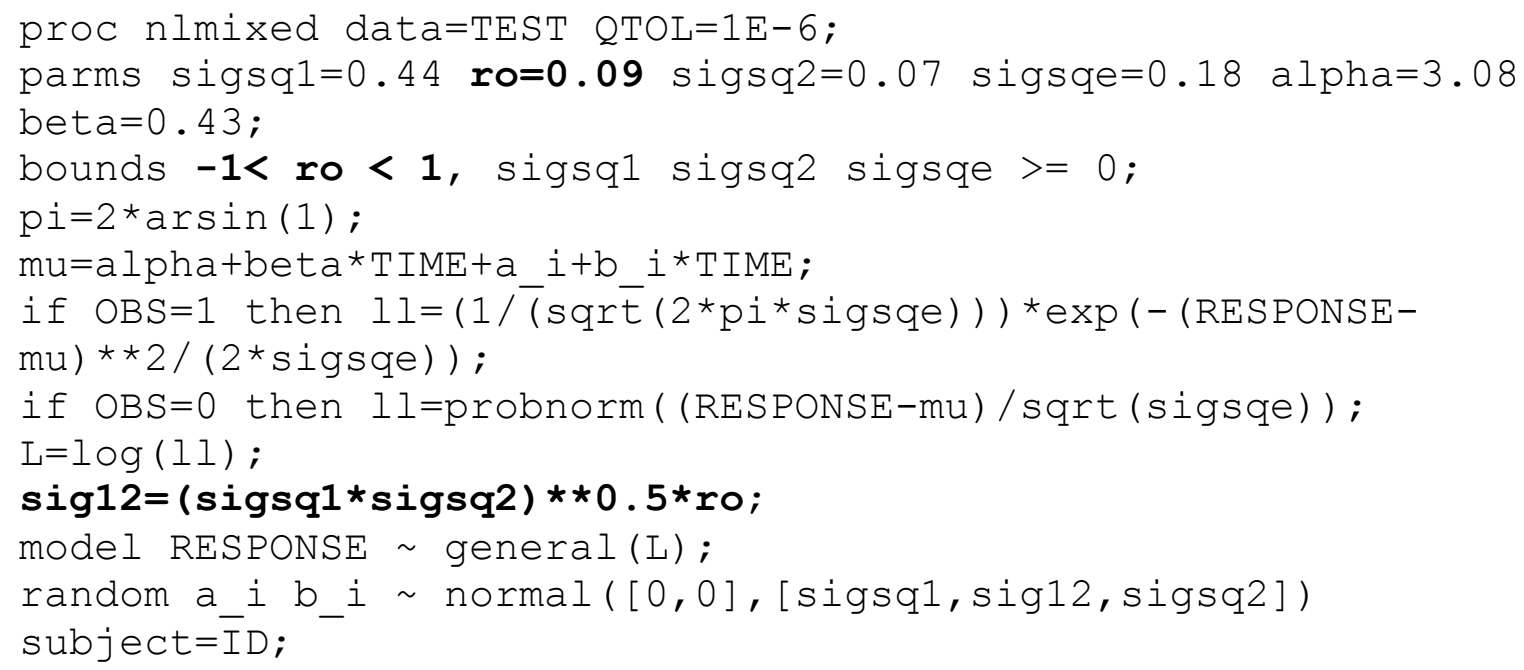

\subsection{Bivariate mixed model}

Extension to a bivariate random effects model as proposed previously with the procedure MIXED [21] is also possible with NLMIXED. Thus, parameters of a bivariate model accounting for left-censoring of one or both markers can be estimated. The key idea is to distinguish the two markers using an indicator (noted VAR in the code). Presentation of dataset must be performed as previously reported [21] with, in addition, an indicator of the censoring status of the measure. The code for a bivariate model including four random effects, i.e. one intercept and one slope for each marker, is presented below. In the programming statement, conditional expectancies given random effects are defined according to the marker:

$$
\left\{\begin{aligned}
E\left(Y_{i j}^{1} \mid a_{i}^{1}, b_{i}^{1}\right) & =\alpha^{1}+\beta^{1} t_{i j}^{1}+a_{i}^{1}+b_{i}^{1} t_{i j}^{1} \\
E\left(Y_{i j}^{2} \mid a_{i}^{2}, b_{i}^{2}\right) & =\alpha^{2}+\beta^{2} t_{i j}^{2}+a_{i}^{2}+b_{i}^{2} t_{i j}^{2}
\end{aligned}\right.
$$

When the covariance matrix of random effects, defined in the statement random, is unstructured, ten variance parameters must be estimated: 
$\left[\begin{array}{l}a_{i}^{1} \\ b_{i}^{1} \\ a_{i}^{2} \\ b_{i}^{2}\end{array}\right] \sim N\left[\left(\begin{array}{l}0 \\ 0 \\ 0 \\ 0\end{array}\right),\left(\begin{array}{llll}s 1 & & & \\ s 2 & s 3 & & \\ s 4 & s 5 & s 6 & \\ s 7 & s 8 & s 9 & s 10\end{array}\right)\right]$

So, model parameters are estimated with this code:

proc nlmixed data=TEST;

parms alphal=0.1 betal=0.1 alpha2=0.1 beta2=0.1 s $1=0.1$ s $2=0.1$ s $3=0.1$ s $4=0.1$ s $5=0.1$ s $6=0.1$ s7=0.1 s $8=0.1$ s $9=0.1$ s $10=0.1$ sce $1=0.1$ sce $2=0.1$;

bounds s1 s3 s6 s10 sce1 sce $2>=0$;

pi=2*arsin (1);

if $\operatorname{VAR}=1$ then do;

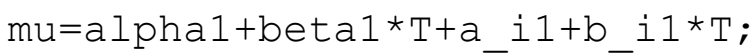

sce $=$ sce 1 ;

end;

else if $V A R=2$ then do;

$\mathrm{mu}=\mathrm{alpha} 2+$ beta $2 * \mathrm{~T}+\mathrm{a} \_\mathrm{i} 2+\mathrm{b} \_\mathrm{i} 2 * \mathrm{~T}$;

$\mathrm{sce}=\mathrm{sce} 2$;

end;

if $\mathrm{OBS}=1$ then $\mathrm{L}=(1 /(\operatorname{sqrt}(2 * \mathrm{pi} * \mathrm{sce}))) * \exp (-(\mathrm{Y}-\mathrm{mu}) * \star 2 /(2 * \mathrm{Sce}))$;

if $\mathrm{OBS}=0$ then $\mathrm{L}=$ probnorm $((\mathrm{Y}-\mathrm{mu}) / \operatorname{sqrt}(\mathrm{sce}))$;

$11=\log (\mathrm{L})$;

model $\mathrm{Y} \sim$ general (ll);

random a_i1 a_i2 b_il b_i2

normal $([\overline{0}, 0,0,0],[\bar{s} 1, s 2, s 3, s 4, s 5, s 6, s 7, s 8, s 9, s 10])$ subject=id; 


\section{Example}

Estimations of parameters for the model (3) were compared according to the two programs previously described. Estimations from a crude approach using the procedure MIXED where left-censored measures were replaced by the value of the threshold were also presented. We used the data set simulated by Lyles et al. [10]. It is available at http://www.blackwellpublishers.co.uk/rss/Volumes/Cv49p4.htm. The true parameters were $\mathrm{N}=50, n_{i}=5, \alpha=3, \beta=0.5, \sigma_{1}^{2}=0.5, \sigma_{2}^{2}=0.1, \sigma_{12}=-0.1$ according to the notations in section 3.1. In the simulated data set, there were 38/250 (15.2\%) left-censored measures of HIV RNA. Results of parameter estimations are reported in table 2. Crude approach without handling left-censoring of HIV RNA leads to biased estimates: especially the fixed slope is underestimated by $14 \%$ and the covariance between intercept and random slope is underestimated by $74 \%$ compared to less than $10 \%$ with other approaches. Obviously, the standard errors of estimates are underestimated with the crude approach because of the simple imputation of the limit of detection for censored measures. The comparison of crude approaches and methods taking into account left-censoring were performed more formally elsewhere [7]. Results according to the method used to take into account left-censoring were similar. Computation times were several seconds for CENSAD and NLMIXED. Analyses were performed in a Windows environment with Pentium III processor. NLMIXED methods were performed using SAS® 8.2. CENSAD has been compiled using Fortran Powerstation $4.0 ®$ with default options. The two methods started with estimations obtained from the crude approach. 


\section{Conclusion}

We presented two approaches to fit linear mixed models accounting for left-censoring of the response and we showed with an example that they gave the same results. Thus, in a practical point of view, to fit mixed models for left-censored repeated measures, one can choose between NLMIXED and CENSAD. The main elements to choose between approaches are the structure of the data and the model used. In fact, CENSAD will be limited when numerous measures are censored while too many random effects will limit NLMIXED because of the numerical integration. Another point is the potential extension of the estimation to more general model. CENSAD allows including a Gaussian process in the error term like a first order auto-regressive process or a Brownian motion. The extension to a bivariate model is direct with NLMIXED (see section 3.2) and possible using the other method [22]). Using NLMIXED, the main limitation is then the number of random effects. In our experience, the procedure was reliable until four random effects leading, for example, to a bivariate model with two intercepts and two random slopes.

Using the approaches presented in this paper, one must keep in mind the limits of the methods. In particular, the models are fully parametric and assume the normality of outcome distribution. Moreover, in case of missing data, estimations are reliable only if the missngness process is not informative [10].

In conclusion, linear mixed models may be estimated accounting for repeated left-censored measures using available tools. In the context of HIV infections, this could be very useful because of the occurrence of longitudinal analyses of HIV RNA evolution and the bias induced by naïve approaches $[6,7,10]$. 


\section{References}

[1] S-PLUS Programmer's Manual, (Statistical Sciences Inc., Seattle, WA, 1991).

[2] W.R. Gilks, A. Thomas, D.J. Spiegelhalter, A language and program for complex Bayesian modelling, J R Stat Soc D 43 (1994) 169-178.

[3] R.C. Littell, G.A. Milliken, W.W. Stroup, R.D. Wolfinger, SAS System for Mixed Models, (SAS Institute, Cary, NC, 1996).

[4] A. Singh, J. Nocerino, Robust estimation of mean and variance using environmental data sets with below detection limit observations, Chemometrics Intell Lab Syst 60 (2002) 69-86. [5] L.H. Moulton, N.A. Halsey, A mixture model with detection limits for regression analyses of antibody response to vaccine, Biometrics 51 (1995) 1570-1578.

[6] J.P. Hughes, Mixed effects models with censored data with application to HIV RNA levels, Biometrics 55 (1999) 625-629.

[7] H. Jacqmin-Gadda, R. Thiébaut, G. Chêne, D. Commenges, Analysis of left-censored longitudinal data with application to viral load in HIV infection, Biostatistics 1 (2000) 355368.

[8] W.B. Paxton, R.W. Coombs, M.J. McElrath et al., Longitudinal analysis of quantitative virologic measures in human immunodeficiency virus-infected subjects with $>$ or $=400 \mathrm{CD} 4$ lymphocytes: implications for applying measurements to individual patients. National Institute of Allergy and Infectious Diseases AIDS Vaccine Evaluation Group, J Infect Dis 175 (1997) 247-254.

[9] I.C. Marschner, R.A. Betensky, V. DeGruttola, S.M. Hammer, D.R. Kuritzkes, Clinical trials using HIV-1 RNA-based primary endpoints: Statistical analysis and potential biases, J Acquir Immune Defic Syndr Hum Retrovirol 20 (1999) 220-227. 
[10] R.H. Lyles, C.M. Lyles, D.J. Taylor, Random regression models for human immunodeficiency virus ribonucleic acid data subject to left censoring and informative dropouts, J R Stat Soc C 49 (2000) 485-497.

[11] H.S. Lynn, Maximum likelihood inference for left-censored HIV RNA data, Stat Med 20 (2001) 33-45.

[12] SAS Institute Inc. The NLMIXED Procedure. SAS/STAT User's Guide, Version 8. Carry, NC: SAS Institute Inc., 2000:2419-2504.

[13] N.M. Laird, J.H. Ware, Random-effects models for longitudinal data, Biometrics 38 (1982) 963-974.

[14] D.W. Marquardt, An algorithm for least squares estimation of nonlinear parameters, J Soc Indust Appl Math 11 (1963) 431-441.

[15] A. Genz, Numerical computation of multivariate normal probabilities, J Comput Graph Statist 1 (1992) 141-149.

[16] A. Genz, Comparison of methods for the computation of multivariate normal probabilities, Comput Sci Stat 25 (1993) 400-413.

[17] R.D. Wolfinger. Fitting non linear mixed models with the new NLMIXED procedure. 24th SAS User Group International Conference. Miami Beach, Florida. 1999.

[18] R. Flechter, Practical methods of optimization, (Wiley, Bath, 1987).

[19] J.C. Pinheiro, D.M. Bates, Approximations to the Log-likelihood Function in the Nonlinear Mixed-effects Model, J Comput Graph Statist 4 (1995) 12-35.

[20] E. Lesaffre, B. Spiessens, On the effect of the number of quadrature points in a logistic random-effects model: An example, J R Stat Soc C 50 (2001) 325-335.

[21] R. Thiébaut, H. Jacqmin-Gadda, G. Chêne, C. Leport, D. Commenges, Bivariate linear mixed models using SAS proc MIXED, Comput Methods Programs Biomed 69 (2002) 249256. 
[22] R. Thiébaut, H. Jacqmin-Gadda, C. Leport et al., Bivariate longitudinal model for the analysis of the evolution of HIV RNA and CD4 cell count in HIV infection taking into account left censoring of HIV RNA measures, J Biopharm Stat 13 (2003) 271-282. 
Table 1. Summary of different presentations to fit a linear mixed model for left-censored repeated gaussian data.

\begin{tabular}{|c|c|c|c|}
\hline Approach & Tool & Integration & Optimisation algorithm \\
\hline $\begin{array}{l}\text { Jacqmin-Gadda et al. } \\
\text { (CENSAD) [7] }\end{array}$ & Fortran 77 & $\begin{array}{l}\text { - Over censored measures } \\
\text { - Subregion adaptative SADMVN } \\
{[15]}\end{array}$ & Marquardt \\
\hline Procedure NLMIXED & $\begin{array}{l}\text { Procedure SAS® /STAT } \\
\text { module }\end{array}$ & $\begin{array}{l}\text { - Over random effects } \\
\text { - Adaptative Gaussian quadrature or } \\
\text { others* }\end{array}$ & Quasi-Newton or others* \\
\hline
\end{tabular}

* See text: 2.3 Computation 
Table 2. Parameters estimation and standard deviation (sd) of a mixed model with one intercept and one slope according to the method used.

Simulated data from Lyles et al. [10]: $\mathrm{N}=50, n_{i}=5, \alpha=3, \beta=0.5, \sigma_{1}^{2}=0.5, \sigma_{2}^{2}=0.1, \sigma_{12}=-0.1, \sigma_{e}^{2}=0.2$.

\begin{tabular}{lcccccc}
\hline Method & $\begin{array}{c}\hat{\alpha} \\
(\mathrm{sd})\end{array}$ & $\begin{array}{c}\hat{\beta} \\
(\mathrm{sd})\end{array}$ & $\begin{array}{c}\hat{\sigma}_{1}^{2} \\
(\mathrm{sd})\end{array}$ & $\begin{array}{c}\hat{\sigma}_{12} \\
(\mathrm{sd})\end{array}$ & $\begin{array}{c}\hat{\sigma}_{2}^{2} \\
(\mathrm{sd})\end{array}$ & $\begin{array}{c}\hat{\sigma}_{e} \\
(\mathrm{sd})\end{array}$ \\
\hline $\begin{array}{l}\text { Censoring not handled } \\
\text { SAS® Proc MIXED) }\end{array}$ & $3.08(0.10)$ & $0.43(0.05)$ & $0.44(0.11)$ & $-0.026(0.042)$ & $0.066(0.029)$ & $0.18(0.02)$ \\
SAS® Proc NL MIXED & $2.94(0.13)$ & $0.51(0.062)$ & $0.66(0.17)$ & $-0.11(0.066)$ & $0.089(0.040)$ & $0.23(0.029)$ \\
CENSAD & $2.94(0.13)$ & $0.50(0.062)$ & $0.66(0.17)$ & $-0.11(0.066)$ & $0.089(0.040)$ & $0.23(0.029)$ \\
\hline
\end{tabular}

